\title{
INVESTIGACIONES
}

\section{Educación para la ciudadanía e identidad en los museos de Estados Unidos. Análisis desde la perspectiva de la educación patrimonial*}

\author{
Citizenship education and identity in U.S. museums. \\ An analysis from a heritage education perspective \\ José Cuenca López, ${ }^{a}$ Myriam Martín Cáceres, ${ }^{a b}$ Daniel Schugurensky ${ }^{c}$ \\ ${ }^{a}$ Facultad de Ciencias de la Educación. Universidad de Huelva \\ Telf.: (34) 959219263. Correo electrónico: jcuenca@uhu.es \\ bTelf.: (34) 959219386. Correo electrónico: myriam.martin@ddcc.uhu.es \\ cArizona State University \\ Correo electrónico: dschugur@asu.edu
}

\begin{abstract}
RESUMEN
Este trabajo analiza las relaciones entre educación para la ciudadanía, identidad y patrimonio, centrándose en el tratamiento didáctico que se desarrolla en varios tipos de museos, en este caso, dentro del ámbito estadounidense. Para ello se analizan, empleando una metodología cualitativa de observación y registro de datos, las propuestas educativas de 15 museos de diferentes características y tipos en los estados de Arizona, California y Nueva York. El análisis de los datos fue guiado por tres categorías conceptuales y 14 subcategorías, cada cual con indicadores específicos. Se ponen de manifiesto diversos aspectos de carácter identitario, en función de las características patrimoniales, institucionales y culturales asociadas a los ámbitos socioespaciales.
\end{abstract}

Palabras clave: identidad, educación patrimonial, museos, educación ciudadana, Estados Unidos.

\section{ABSTRACT}

This paper analyzes the relationship between citizenship education, identity and heritage, focusing on the pedagogical interventions undertaken in a variety of museums, in this case, within the United States. For this purpose, the educational proposals of 15 museums of different characteristics and types in the states of Arizona, California and New York are analyzed, using a qualitative methodology for data collection. The data analysis was guided by three conceptual categories and 14 sub-categories, each one with specific indicators. Various aspects of identity are revealed, depending on a variety of cultural, institutional, social and geographical features of the museums and their contexts.

Keywords: identity, heritage education, museums, citizenship education, United States.

\footnotetext{
* Este estudio ha sido posible gracias a los equipos de trabajo y a la financiación proporcionada por el proyecto I+D+i Educación patrimonial para la inteligencia territorial y emocional de la ciudadanía. Análisis de buenas prácticas, diseño e intervención en la enseñanza obligatoria (EDU2015-67953-P) (MINECO/FEDER) y la RED14: Red de investigación en enseñanza de las Ciencias Sociales (EDU2014-51720-REDT), concedidos por el Ministerio de Economía y Competitividad, así como por las subvenciones del Subprograma de movilidad en el marco del Plan Estatal de Investigación Científica y Técnica y de Innovación 2013-2016 en I+D+i (Ministerio de Educación, Cultura y Deporte).
} 


\section{INTRODUCCIÓN}

En un libro de reciente publicación, John Falk (2016) afirmó que la clave para entender la experiencia de los visitantes a un museo es la construcción de la identidad. En la misma línea de pensamiento, hace más de dos décadas Carol Duncan (1994) señaló que los museos podrían ser considerados como poderosas maquinarias para la definición de las identidades. Según ella, controlar un museo significa controlar la representación que una comunidad tiene de sí misma y la forma en que se presenta a otras comunidades. Esto también incluye el poder para definir y jerarquizar a diferentes grupos humanos, así como para establecer qué grupos tienen más importancia en el patrimonio de la comunidad y, por tanto, en su identidad.

La identidad es una dimensión central de la ciudadanía, y se refiere a los sentimientos de pertenencia a una o más comunidades políticas. En las instituciones educativas escolares de Estados Unidos la identidad suele entenderse como lealtad al estado nación, y se expresa tanto en contenidos curriculares como en una serie de rituales que incluyen el izamiento de la bandera y el canto del himno nacional y otras canciones patrias (Schugurensky 2016). Estos aprendizajes se refuerzan socialmente en la educación informal cotidiana (pedagogía pública) expresada, por ejemplo, en monumentos, festejos por efemérides, desfiles, homenajes a personajes considerados heroicos, películas, desfiles, programas de televisión, revistas, eventos deportivos, y también en los museos, la institución que nos ocupa en este trabajo.

Así como la identidad nacional se relaciona con sentimientos de pertenencia e inclusión, también se puede relacionar (por acción u omisión) con sentimientos de exclusión y rechazo. Los museos, como otras instituciones educativas, pueden presentar el patrimonio de diferentes maneras, desde diferentes perspectivas y con diferentes intencionalidades pedagógicas. Por ejemplo, el patrimonio puede presentarse de una manera rígida y acrítica (como algo 'dado') o como algo construido socialmente que puede problematizarse y analizarse críticamente. Este artículo explora justamente estas dinámicas en 15 museos de Estados Unidos de diferentes tamaños, recursos y temáticas utilizando tres grandes categorías analíticas. La primera es el concepto de patrimonio que subyace en la propuesta museística. La segunda es el modelo de comunicación patrimonial que orienta la tarea pedagógica del museo. La tercera categoría se enfoca en la relación entre patrimonio e identidad. Estas tres categorías incluyen 14 subcategorías que se sistematizan a través de una serie de indicadores.

\section{MARCO TEÓRICO}

Los últimos años están suponiendo una clara consolidación de la línea de investigación de la educación patrimonial en los entornos de enseñanza y aprendizaje de carácter formal, no formal e informal. Así se pone de manifiesto por los últimos monográficos de revistas especializadas como Patrimonio Cultural de España (número 5 de 2011), Clío. History and History Teaching (número 40 de 2014), Educatio siglo XXI (número 33.1 de 2015), Revista de Educación (número 375 de 2017). Igualmente, se constata en los continuos congresos que sobre esta temática se están desarrollando, entre los que destacan las tres ediciones, hasta el momento, del Congreso Internacional de Educación Patrimonial celebradas en Madrid en 2012, 2014 y 2016. No se puede olvidar la redacción y puesta en marcha del Plan 
Nacional de Educación y Patrimonio, desarrollado por el Instituto de Patrimonio Cultural de España, perteneciente al Ministerio de Educación, Cultura y Deporte (Fontal \& Ibáñez, 2015). Recientes trabajos llevados a cabo por Fontal e Ibáñez (2017) y por Martín-Cáceres y Cuenca (2015) recogen rigurosas actualizaciones de la situación de la investigación en este campo en los últimos años, en el panorama español e internacional, centrándose en el impacto de los estudios desarrollados que permiten una aproximación muy certera a lo realizado hasta ahora, y que posibilitan una proyección hacia las líneas de actuación futuras (Cuenca \& Estepa, 2013).

Así, se han desarrollado enormemente los estudios de museos desde múltiples perspectivas (Macdonald, 2011), siendo muy destacable la producción investigadora en el ámbito de la educación no formal, abordando análisis de las propuestas didácticas realizadas en museos o centros de interpretación del patrimonio (Calaf \& Suárez, 2015; Estepa, 2013).

Aunque son más escasos los trabajos que relacionan patrimonio, identidad y ciudadanía, podemos resaltar algunos. Entre ellos, son de gran interés las reflexiones y estudios sobre patrimonio, paisaje y ciudadanía de Brusa (2011), o los de Semedo (2007) y Pinto (2016), donde se trabaja aspectos relacionados con museos, público y ciudadanía, así como los que conectan comunicación, museo e identidad (Copeland, 2009; Davis, 2007; Falk, Dierking \& Adams, 2011; Martín-Cáceres \& Cuenca, 2011; Semedo, 2015). En la misma línea, las relaciones entre los ámbitos formales, no formales e informales en la educación patrimonial y sus conexiones con el campo de las escalas identitarias han sido objeto de estudio por Calaf (2010), Fontal (2013) o Molina, Llonch y Martínez (2016), siempre trabajándose desde perspectivas interdisciplinares. A ello se unen los trabajos que abordan la evaluación de los programas educativos en museos y conjuntos patrimoniales (Cuenca et al., 2014; Fontal \& Gómez-Redondo, 2016; Ibáñez et al., 2012; Vicent, Ibáñez \& Asensio, 2015), aspectos a los que cada vez se les está otorgando una mayor relevancia en el campo de la investigación en educación patrimonial.

A partir de aquí, el marco de referencia teórico desde el que se desarrolla este estudio pasa por considerar las conexiones directas entre patrimonio, identidad y ciudadanía. Así, se establece una visión del patrimonio y de la educación patrimonial de carácter sistémico, participativo, interactivo, complejo y sociocrítico. Los referentes iniciales de esta perspectiva teórica parten de la visión del concepto de patrimonio desde una perspectiva holística y sistémica. Para la definición de patrimonio se parte de múltiples visiones: antropológica, temporal y sociocrítica, a lo que se une las relaciones identitarias (MartínCáceres \& Cuenca, 2016).

Con las propuestas de educación patrimonial se trata de superar las barreras disciplinares, metodológicas y curriculares para que el patrimonio aporte todo su caudal educativo a la formación de la ciudadanía. Desde esta visión, los elementos patrimoniales se articulan como un único hecho sociocultural constituido, de manera holística, por diversas manifestaciones de carácter histórico, artístico, etnológico, científico-tecnológico y medioambiental, que en conjunción permiten el conocimiento integral de las diferentes sociedades tanto del pasado como del presente, dando lugar a estructuras de identidad social que se convierten en símbolos culturales que permiten desarrollar visiones interculturales en la sociedad (Martín-Cáceres, 2012).

De esta manera, la didáctica del patrimonio no constituye un fin en sí mismo, sino que debe integrarse en el proceso educativo, dentro de las grandes metas establecidas 
para la educación, particularmente en la formación de la ciudadanía. A través de los referentes patrimoniales se puede potenciar el conocimiento reflexivo de la realidad, independientemente de que ello conlleve objetivos relacionados con la propia conservación y valoración del patrimonio, así como con el propio conocimiento de dichos referentes y sus procedimientos de análisis e investigación, que no han de confundirse con el fin último de este proceso educativo (Cuenca \& Martín-Cáceres, 2014).

\section{METODOLOGÍA}

Este trabajo se articula desde premisas de investigación de corte cualitativo (Gibbs, 2012), desarrollándose a partir de la comprensión e interpretación del significado de los fenómenos sociales, la explicitación de los valores del investigador, la asunción de la necesidad de la integración entre teoría y práctica y el subjetivismo reconocido (León \& Montero, 2002; Wiersma, 2000). De esta manera, se analizan las propuestas de educación patrimonial en los museos y espacios de comunicación patrimonial seleccionados. Así, el objetivo de este estudio consiste en comprobar el tratamiento de la identidad y la ciudadanía como parte de los contenidos educativos de los museos, de manera que podamos acercarnos al conocimiento de las conexiones patrimonio identidad ciudadanía que se producen en estos centros, sin intervenir directamente en la realidad. El trabajo se desarrolla como observadores externos, en lo referente a la proyección del patrimonio y todo lo que este conlleva, desde una visión simple hasta otra más compleja (Álvarez-Gayou, 2003; Flick, 2004), con el fin de evaluar los programas socioeducativos desde la visión de la educación patrimonial (San Fabián, 2014).

Las instituciones que participan como informantes en este estudio consisten en 15 museos del ámbito histórico artístico, situados en los Estados Unidos de América, concretamente en las ciudades de Phoenix, Los Angeles y New York (Tabla 1). Se han seleccionado estas localidades por su carácter metropolitano y el alto nivel de inmigración (siguiendo los datos proporcionados por United States Census Bureau correspondientes a 2017), y por consiguiente de relaciones de interculturalidad que existe en ellas, aspecto que liga directamente con los intereses del estudio que aquí se presenta. New York (8.175.133) y Los Angeles (3.792.621) son las dos ciudades más pobladas de Estados Unidos, con unos índices de inmigración de 22,9 y 27,3\% respectivamente en sus correspondientes estados, y Phoenix la sexta (1.445.632), y, aunque el índice de inmigración es algo menor $(13,4 \%$ en el estado de Arizona), el interés de su situación geográfica fronteriza con México y la complejidad de sus relaciones culturales la convertían también en una ciudad relevante para este estudio.

La selección de los centros elegidos en estas ciudades atiende al criterio de contar en el estudio con diversos tipos de museos, en función de sus dimensiones y características de los materiales que conservan y exponen. Así, en la muestra existen museos de grandes dimensiones y reconocimiento internacional, con fondos de amplio espectro y procedencia, junto a otros centros de ámbito local y cuyo objeto de trabajo se centra en su contexto cercano. De esta manera se pretende determinar las diferencias existentes entre unos centros y otros, respecto a su tratamiento en las propuestas de educación patrimonial que se establecen, con el objeto de comprobar si estas instituciones patrimoniales desarrollan programas educativos tendientes a la reflexión sobre los problemas identitarios y la conformación de una ciudadanía crítica. 
Tabla 1. Instituciones informantes del estudio

\begin{tabular}{|c|c|c|c|}
\hline Estado & Ciudad & Museo & Características \\
\hline \multirow{4}{*}{ Arizona } & \multirow{4}{*}{ Phoenix } & Heard Museum & Gran museo nacional histórico y etnológico \\
\hline & & Musical Instrument Museum & Gran museo nacional histórico, artístico y etnológico \\
\hline & & Phoenix Art Museum & Gran museo local artístico \\
\hline & & $\begin{array}{l}\text { Pueblo Grande Museum and } \\
\text { Archaeological Park }\end{array}$ & Pequeño museo local histórico-arqueológico \\
\hline \multirow{4}{*}{ California } & \multirow{4}{*}{ Los Angeles } & Getty Museum & Gran museo internacional, histórico-artístico \\
\hline & & $\begin{array}{l}\begin{array}{l}\text { Los Angeles County Museum } \\
\text { of Arts }\end{array} \\
\end{array}$ & Gran museo internacional, histórico-artístico \\
\hline & & \begin{tabular}{|l} 
Los Angeles Museum of \\
Holocaust
\end{tabular} & Pequeño museo local histórico \\
\hline & & Museum of Tolerance & Gran museo nacional histórico y etnológico \\
\hline \multirow{7}{*}{ New york } & \multirow{7}{*}{ New york } & 9-11 Memorial Museum & Pequeño museo local histórico \\
\hline & & Guggenheim NY & Gran museo internacional artístico \\
\hline & & Metropolitan Museum of Art & Gran museo internacional histórico-artístico \\
\hline & & Museum of Modern Art & Gran museo internacional histórico-artístico \\
\hline & & Museo de El Barrio & Pequeño museo local artístico \\
\hline & & $\begin{array}{l}\text { Museum of the City of New } \\
\text { York }\end{array}$ & Pequeño museo local histórico y etnológico \\
\hline & & $\begin{array}{l}\text { National Museum of the } \\
\text { American Indian }\end{array}$ & Gran museo nacional histórico-artístico \\
\hline
\end{tabular}

En concreto, para atender al tipo de gran museo de carácter nacional internacional y un número de visitantes muy alto, se han seleccionado 9 museos: Heard Museum y Musical Instrument Museum en Phoenix; Getty Museum, Los Angeles County Museum y Museum of Tolerance en Los Angeles; y Guggenheim NY, Metropolitan Museum of Art, Museum of Modern Art y National Museum of American Indian en New York.

En cuanto a los centros de carácter más local comarcal, se han seleccionado 6 museos: Phoenix Art Museum y Pueblo Grande Museum (Phoenix), Los Angeles Museum of Holocaust y 9-11 Memorial Museum, Museo de El Barrio y Museum of the City of New York (Nueva York).

La información se obtuvo a través de la observación de las salas de exposición de las instituciones museísticas seleccionadas, de los materiales y programas que han diseñado y de las actividades que se realizan por los visitantes en estos espacios. Para ello, el procedimiento de toma de datos ha consistido en la observación y registro a través de un cuaderno de campo y de imágenes de todas las exposiciones permanentes de los diferentes museos objeto de estudio, centrándose en su diseño y en los recursos disponibles en las diversas salas como apoyo a la visita. Igualmente, se han recogido los materiales didácticos que se ponen a disposición del público y que facilitan la interpretación de los 
Estudios Pedagógicos XLIII, N 4: 29-48, 2017

EDUCACIÓN PARA LA CIUDADANÍA E IDENTIDAD EN LOS MUSEOS DE ESTADOS UNIDOS. ANÁLISIS DESDE LA PERSPECTIVA DE LA EDUCACIÓN PATRIMONIAL

contenidos que comunican los museos. Finalmente, dentro del proceso de observación se registra también información sobre actividades desarrolladas por las propias instituciones patrimoniales con diversos tipos de público (infantil, familiar y turístico).

La recogida de información se realizó mediante el diseño y aplicación de unas fichas de observación y registro de datos, y el análisis se desarrolló a través de un sistema de categorías preestablecidas, ya validado por su empleo en otros estudios previos de características similares (Calaf \& Suárez, 2015; Estepa, 2013; Jiménez, Cuenca \& Ferreras, 2010; Martín-Cáceres, 2012; Martín-Cáceres \& Cuenca, 2011).

Así, tras la obtención de datos, toda la información registrada a partir de la observación de las salas y de las actividades realizadas por los visitantes, así como de los materiales didácticos de que disponen, se analiza usando como instrumento de investigación el sistema de categorías (Tabla 2) que estructura el proceso de interpretación de los datos.

Este sistema de categorías se concibe como un instrumento que organiza el contenido y la estructura de las fichas de observación, guiando el proceso analítico de la investigación. Este instrumento se organiza como una hipótesis de progresión (Cuenca, 2002) compuesta por tres niveles de evolución, estableciendo una evolución de los procesos de educación patrimonial desde la conceptualización más simple hasta la más compleja. Se compone de tres categorías de estudio que se incluyen 14 subcategorías y una variedad de indicadores que facilitan la adscripción y la interpretación de las unidades de información en el proceso de análisis de datos, permitiendo abordar los datos recogidos en el trabajo de campo de una forma sistemática y rigurosa.

Tabla 2. Categorías, subcategorías, indicadores y descriptores

\begin{tabular}{|c|c|c|c|}
\hline Categorías & Subcategorías & Indicadores & Descriptores \\
\hline \multirow{13}{*}{$\begin{array}{l}\text { I. Concepto } \\
\text { de patrimonio }\end{array}$} & \multirow{6}{*}{$\begin{array}{l}\text { 1. Perspectivas sobre } \\
\text { el patrimonio }\end{array}$} & $\begin{array}{l}\text { Fetichista- } \\
\text { excepcionalista }\end{array}$ & $\begin{array}{l}\text { Admiración irracional, convirtiéndose en amuleto, objeto } \\
\text { de culto o mágico. Escasez, rareza, singularidad y valor } \\
\text { crematístico. }\end{array}$ \\
\hline & & Monumental & $\begin{array}{l}\text { Grandiosidad y reconocido prestigio de elementos vivos } \\
\text { y no vivos, materiales o inmateriales. }\end{array}$ \\
\hline & & Estética & Belleza natural, artística y estilística. \\
\hline & & Temporal & $\begin{array}{l}\text { Carácter evolutivo-temporal de los cambios en la } \\
\text { naturaleza, especies relictas. }\end{array}$ \\
\hline & & Diversidad & Riqueza en bio-geodiversidad y/o diversidad cultural. \\
\hline & & $\begin{array}{l}\text { Simbólico- } \\
\text { identitaria }\end{array}$ & $\begin{array}{l}\text { Elementos simbólicos que caracterizan a una sociedad, } \\
\text { un entorno natural, la geo-biodiversidad. }\end{array}$ \\
\hline & \multirow{4}{*}{$\begin{array}{l}\text { 2. Tipos de } \\
\text { patrimonio }\end{array}$} & $\begin{array}{l}\text { Patrimonio } \\
\text { natural- } \\
\text { histórico- } \\
\text { artístico }\end{array}$ & $\begin{array}{l}\text { Elementos de carácter medioambiental. Referentes } \\
\text { arqueológicos y documentales. Manifestaciones } \\
\text { correspondientes a los diferentes movimientos } \\
\text { estilísticos. Paisajes asociados a ellos. }\end{array}$ \\
\hline & & $\begin{array}{l}\text { Patrimonio } \\
\text { etnológico }\end{array}$ & $\begin{array}{l}\text { Elementos significativos y tradicionales que explican el } \\
\text { cambio social y paisajes asociados a ellos. }\end{array}$ \\
\hline & & $\begin{array}{l}\text { Patrimonio } \\
\text { científico- } \\
\text { tecnológico }\end{array}$ & $\begin{array}{l}\text { Objetos e instrumentos de la ciencia y componentes } \\
\text { tecnológicos e industriales de relevancia social y paisajes } \\
\text { asociados. }\end{array}$ \\
\hline & & $\begin{array}{l}\text { Patrimonio } \\
\text { holístico }\end{array}$ & $\begin{array}{l}\text { Consideración global e integrada de todas las } \\
\text { manifestaciones anteriores. }\end{array}$ \\
\hline & \multirow{3}{*}{$\begin{array}{l}\text { 3. Nivel de } \\
\text { disciplinariedad }\end{array}$} & Unidisciplinar & Se trabaja una sola tipología patrimonial. \\
\hline & & Multidisciplinar & Se trabajan varios tipos de patrimonio de forma sumativa. \\
\hline & & Interdisciplinar & Se trabajan varios tipos de patrimonio de forma sistémica. \\
\hline
\end{tabular}




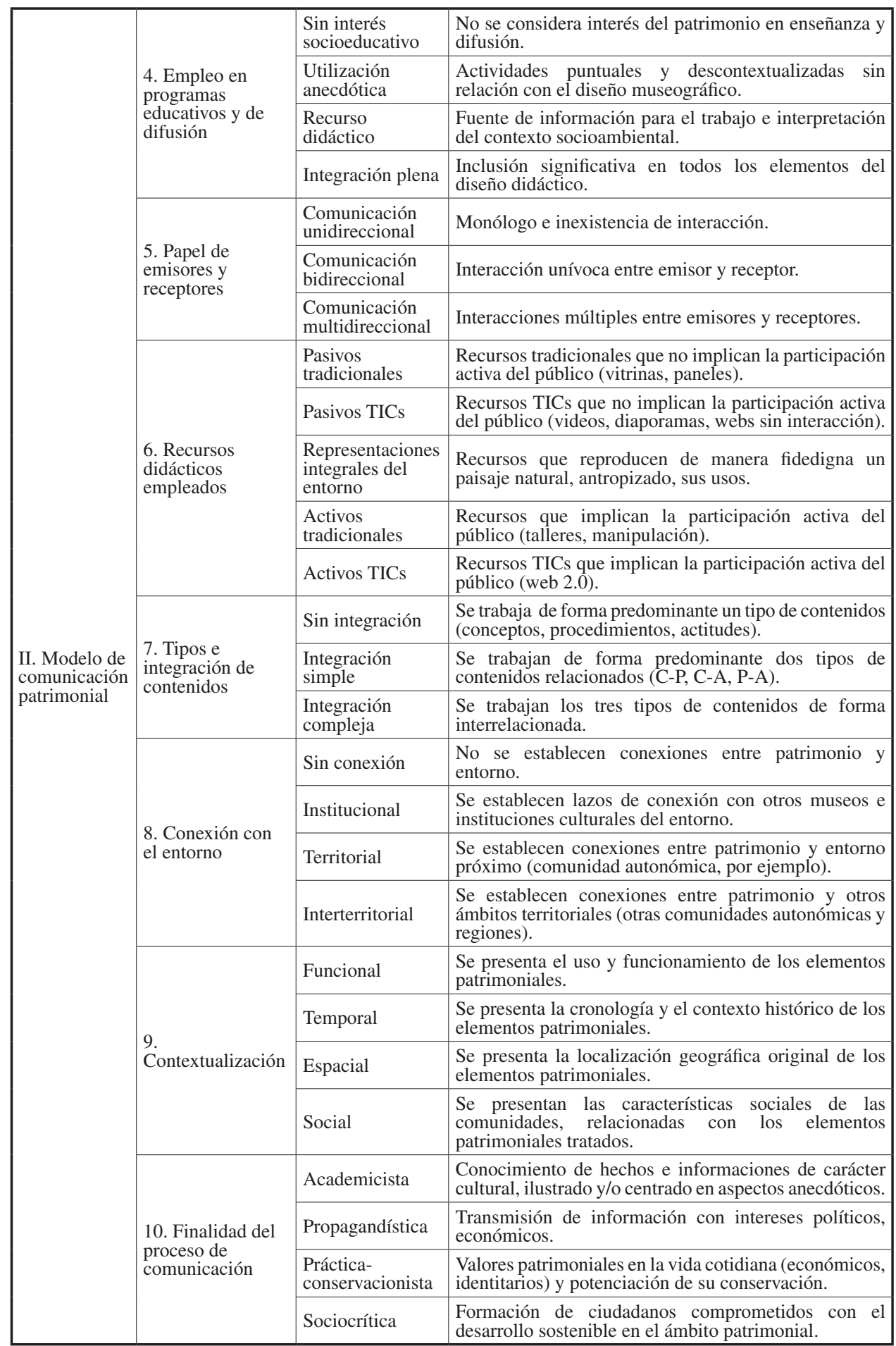




\begin{tabular}{|c|c|c|c|}
\hline \multirow{15}{*}{$\begin{array}{l}\text { III. Patrimonio } \\
\text { e identidad }\end{array}$} & \multirow{3}{*}{$\begin{array}{l}\text { 11. Escalas de } \\
\text { identidad }\end{array}$} & Individual & $\begin{array}{l}\text { Reconocimiento del valor simbólico e identitario exclusi- } \\
\text { vamente de aquellos elementos patrimoniales cercanos y } \\
\text { directamente relacionados con el individuo por su expe- } \\
\text { riencia personal. }\end{array}$ \\
\hline & & Social & $\begin{array}{l}\text { Además del anterior, reconocimiento del valor simbólico } \\
\text { e identitario de elementos patrimoniales relativos a la } \\
\text { cultura propia. }\end{array}$ \\
\hline & & Poliidentidad & $\begin{array}{l}\text { Además de los anteriores, reconocimiento del valor sim- } \\
\text { bólico e identitario de elementos patrimoniales externos } \\
\text { a la propia cultura. }\end{array}$ \\
\hline & \multirow{5}{*}{$\begin{array}{l}\text { 12. Tipología } \\
\text { patrimonial e } \\
\text { identidad }\end{array}$} & Etnológico & $\begin{array}{l}\text { Identificación con elementos significativos y tradicionales } \\
\text { y paisajes asociados. }\end{array}$ \\
\hline & & Natural & Identificación con elementos de carácter medioambiental. \\
\hline & & Histórico & $\begin{array}{l}\text { Identificación con referentes arqueológicos y documen- } \\
\text { tales. }\end{array}$ \\
\hline & & Artístico & $\begin{array}{l}\text { Identificación con manifestaciones de los diferentes mo- } \\
\text { vimientos estilísticos. }\end{array}$ \\
\hline & & Holístico & $\begin{array}{l}\text { Identificación indiferenciada con diversas manifestacio- } \\
\text { nes patrimoniales. }\end{array}$ \\
\hline & \multirow{3}{*}{$\begin{array}{l}\text { 13. Representaciones } \\
\text { identitarias }\end{array}$} & Aidentitario & $\begin{array}{l}\text { No se comunica ningún referente de identificación } \\
\text { cultural. }\end{array}$ \\
\hline & & Estereotipos & $\begin{array}{l}\text { Comunicación de elementos patrimoniales que fomentan } \\
\text { una visión reduccionista y falseada de la cultura. }\end{array}$ \\
\hline & & $\begin{array}{l}\text { Señas de } \\
\text { identidad }\end{array}$ & $\begin{array}{l}\text { Comunicación de elementos patrimoniales como } \\
\text { símbolos sociales que aportan una visión significativa de } \\
\text { la cultura. }\end{array}$ \\
\hline & \multirow{4}{*}{$\begin{array}{l}\text { 14. Elementos } \\
\text { constitutivos de la } \\
\text { identidad/patrimonio }\end{array}$} & $\begin{array}{l}\text { Afectivo- } \\
\text { emocionales }\end{array}$ & $\begin{array}{l}\text { Relaciones sociales (familia, amigos, asociaciones) y } \\
\text { referentes patrimoniales asociados a ellas (elementos } \\
\text { festivos, gastronomía, útiles de la vida cotidiana). }\end{array}$ \\
\hline & & Territoriales & $\begin{array}{l}\text { Elementos naturales, espaciales y arquitectónicos y } \\
\text { paisajes asociados a hechos socio-históricos. }\end{array}$ \\
\hline & & $\begin{array}{l}\text { Convenciones } \\
\text { sociopolíticas }\end{array}$ & $\begin{array}{l}\text { Elementos sociopolíticos convencionales (bandera, him- } \\
\text { no, escudos heráldicos) y todos los referentes patrimo- } \\
\text { niales tipificados en la legislación. }\end{array}$ \\
\hline & & $\begin{array}{l}\text { Multiplicidad } \\
\text { identitaria }\end{array}$ & $\begin{array}{l}\text { Integración de todos los elementos anteriores y valoración } \\
\text { y respeto a la diversidad de identidades. }\end{array}$ \\
\hline
\end{tabular}

Fuente: elaboración propia.

La categoría I aborda el "Concepto de patrimonio" que se encuentra presente en las propuestas comunicativas planteadas en los museos seleccionados. Las variables que la componen abordan la perspectiva sobre la consideración del patrimonio, las diversas tipologías patrimoniales y el nivel de interdisciplinariedad que se desarrolla en las diferentes propuestas, en función de las diferentes temáticas que las instituciones museísticas desarrollan en cada caso. En la categoría II ("Modelo de comunicación patrimonial") se analiza el tratamiento didáctico que los museos estudiados planifican con respecto a los elementos patrimoniales que conservan, valorando el interés educativo del patrimonio; el papel que juegan en las propuestas didácticas el profesorado, los gestores, el alumnado y el público; los recursos y materiales empleados como apoyo a las diferentes experiencias; los tipos de contenidos trabajados, su integración y contextualización; la conexión de las propuestas con el entorno y las finalidades de todos los procesos educativos. La tercera categoría, "Patrimonio e Identidad", se centra en el análisis de las relaciones que 
las propuestas educativas de los museos establecen entre estos dos tópicos en relación con el conocimiento de los elementos patrimoniales constitutivos de la identidad, sus representaciones, las escalas de identidad vinculadas con el patrimonio y las conexiones entre las tipologías patrimoniales y las concepciones de identidad.

\section{ANÁLISIS DE RESULTADOS}

Este estudio se ha desarrollado a partir del tratamiento de las tres categorías de estudio que se han presentado en el apartado anterior, en las cuales se profundiza en el conocimiento de las características y tipologías patrimoniales que se abordan en los procesos educativos desarrollados en los museos seleccionados. El análisis del tratamiento didáctico que en ellos se lleva a cabo, se realiza mediante el análisis de los modelos educativos que se emplean, y las relaciones entre patrimonio e identidad que se trabajan en ellos, siempre desde la perspectiva de la educación para la ciudadanía, como una de las finalidades que le da sentido al patrimonio como elemento de socialización.

A nivel descriptivo, en la Tabla 3 se presenta la información general obtenida a través de la información en cada uno de los museos, estructurada por categorías de análisis.

Tabla 3. Análisis de museos por categorías

\begin{tabular}{|c|c|c|c|}
\hline Museos & Categoría I & Categoría II & Categoría III \\
\hline $\begin{array}{l}\text { Heard } \\
\text { Museum }\end{array}$ & $\begin{array}{l}\text { Perspectiva estética, temporal } \\
\text { y de diversidad, con conexio- } \\
\text { nes con los grupos sociales } \\
\text { del entorno. } \\
\text { Patrimonio Histórico-artístico } \\
\text { y etnológico. Carácter inter- } \\
\text { disciplinar. }\end{array}$ & $\begin{array}{l}\text { Recurso didáctico. } \\
\text { Comunicación unidireccional, } \\
\text { complementado con talleres y } \\
\text { actividades específicas. } \\
\text { Predominio de recursos pasi- } \\
\text { vos tradicionales y TIC. } \\
\text { Integración compleja de con- } \\
\text { tenidos. } \\
\text { Conexión interterritorial y } \\
\text { contextualización funcional, } \\
\text { temporal, espacial y social. } \\
\text { Finalidad sociocrítica. }\end{array}$ & $\begin{array}{l}\text { Escala de identidad social, de } \\
\text { carácter holística. } \\
\text { Trabaja señas de identidad. } \\
\text { Visiones afectivo-emociona- } \\
\text { les, territoriales y de multipli- } \\
\text { cidad identitaria. }\end{array}$ \\
\hline $\begin{array}{l}\text { Musical } \\
\text { Instrument } \\
\text { Museum }\end{array}$ & $\begin{array}{l}\text { Perspectiva fetichista-excep- } \\
\text { cionalista, estética, temporal, } \\
\text { de diversidad y simbólico- } \\
\text { identitaria. } \\
\text { Patrimonio Histórico-artísti- } \\
\text { co, etnológico, científico-tec- } \\
\text { nológico, desde visiones ho- } \\
\text { lísticas. Carácter interdiscipli- } \\
\text { nar }\end{array}$ & $\begin{array}{l}\text { Recurso didáctico. } \\
\text { Comunicación unidireccional } \\
\text { en salas, multidireccional en } \\
\text { talleres. } \\
\text { Predominio de recursos pasi- } \\
\text { vos tradicionales y TIC. Acti- } \\
\text { vos tradicionales en espacios } \\
\text { de manipulación. } \\
\text { No hay integración de conteni- } \\
\text { dos. } \\
\text { Conexión interterritorial y } \\
\text { contextualización funcional, } \\
\text { temporal, espacial y social. } \\
\text { Finalidad academicista, propa- } \\
\text { gandística y conservacionista. }\end{array}$ & $\begin{array}{l}\text { Escala de identidad social y } \\
\text { poliidentitaria, de carácter } \\
\text { histórico y etnológico. } \\
\text { Trabaja desde una visión este- } \\
\text { reotipada. } \\
\text { Tratamiento de la identidad a } \\
\text { través de las convenciones so- } \\
\text { ciopolíticas. }\end{array}$ \\
\hline
\end{tabular}




\begin{tabular}{|c|c|c|c|}
\hline $\begin{array}{l}\text { Phoenix Art } \\
\text { Museum }\end{array}$ & $\begin{array}{l}\text { Perspectiva excepcionalista, } \\
\text { monumental, estética y tem- } \\
\text { poral. } \\
\text { Patrimonio artístico. } \\
\text { Carácter unidisciplinar }\end{array}$ & $\begin{array}{l}\text { Escaso interés educativo. } \\
\text { Comunicación unidireccional, } \\
\text { con recursos pasivos tradicio- } \\
\text { nales. } \\
\text { Sin integración de contenidos } \\
\text { (solo información). } \\
\text { Sin conexión con el entorno ni } \\
\text { contextualización. } \\
\text { Finalidad academicista y pro- } \\
\text { pagandística. }\end{array}$ & $\begin{array}{l}\text { Discurso aidentitario, desde } \\
\text { las perspectivas de las relacio- } \\
\text { nes patrimonio-identidad, tan- } \\
\text { to en lo referido a las escalas, } \\
\text { representaciones y constitu- } \\
\text { ción, aunque siempre están } \\
\text { presentes los aspectos artísti- } \\
\text { cos. }\end{array}$ \\
\hline $\begin{array}{l}\text { Pueblo Grande } \\
\text { Museum and } \\
\text { Archaeological } \\
\text { Park }\end{array}$ & $\begin{array}{l} \\
\text { Perspectiva temporal. } \\
\text { Patrimonio Histórico-arqueo- } \\
\text { lógico. Carácter unidisciplinar }\end{array}$ & \begin{tabular}{|l|} 
Recurso didáctico. \\
Comunicación unidireccional, \\
multidireccional en talleres. \\
Predominio de recursos pasi- \\
vos tradicionales y TIC. \\
Activos tradicionales en espa- \\
cios de manipulación. \\
Integración simple de infor- \\
mación, con procedimientos \\
en talleres. \\
Conexión territorial y contex- \\
tualización funcional, tempo- \\
ral y espacial. \\
Finalidad academicista.
\end{tabular} & $\begin{array}{l}\text { Escala de identidad social, en } \\
\text { conexión con comunidades } \\
\text { indígenas. } \\
\text { Relación identidad con patri- } \\
\text { monio histórico, a partir de } \\
\text { una visión territorial. }\end{array}$ \\
\hline Getty Museum & $\begin{array}{l}\text { Perspectiva excepcionalista, } \\
\text { monumental y estética. } \\
\text { Patrimonio Histórico-artísti- } \\
\text { co. } \\
\text { Carácter unidisciplinar. }\end{array}$ & $\begin{array}{l}\text { Recurso didáctico. } \\
\text { Comunicación unidireccional, } \\
\text { bidireccional a través de uso } \\
\text { de ipod. } \\
\text { Predominio de recursos pasi- } \\
\text { vos tradicionales y TIC Acti- } \\
\text { vos TIC mediante ipod. } \\
\text { Integración simple de infor- } \\
\text { mación, con procedimientos a } \\
\text { través de pantallas virtuales. } \\
\text { Conexión interterritorial y } \\
\text { contextualización funcional, } \\
\text { temporal, espacial y social. } \\
\text { Finalidad academicista y pro- } \\
\text { pagandística. }\end{array}$ & $\begin{array}{l}\text { Discurso aidentitario, desde } \\
\text { las perspectivas de las relacio- } \\
\text { nes patrimonio-identidad, tan- } \\
\text { to en lo referido a las escalas, } \\
\text { representaciones, tipologías y } \\
\text { constitución. }\end{array}$ \\
\hline $\begin{array}{l}\text { Los Angeles } \\
\text { County } \\
\text { Museum of } \\
\text { Arts }\end{array}$ & \begin{tabular}{|l|} 
\\
\\
Perspectiva excepcionalista, \\
monumental y estética. \\
Patrimonio Histórico-artísti- \\
co. \\
Carácter unidisciplinar. \\
\end{tabular} & $\begin{array}{l}\text { Uso didáctico anecdótico. } \\
\text { Comunicación unidireccional, } \\
\text { multidireccional en los talle- } \\
\text { res. } \\
\text { Predominio de recursos pasi- } \\
\text { vos. Activos tradicionales a } \\
\text { través de talleres. } \\
\text { Sin integración de contenidos } \\
\text { (solo información), salvo pro- } \\
\text { cedimientos en talleres. } \\
\text { Conexión interterritorial y } \\
\text { contextualización temporal y } \\
\text { espacial. } \\
\text { Finalidad academicista y pro- } \\
\text { pagandística. }\end{array}$ & $\begin{array}{l}\text { Discurso aidentitario, desde } \\
\text { las perspectivas de las relacio- } \\
\text { nes patrimonio-identidad, tan- } \\
\text { to en lo referido a las escalas, } \\
\text { representaciones y constitu- } \\
\text { ción, aunque siempre están } \\
\text { presentes los aspectos artísti- } \\
\text { cos. }\end{array}$ \\
\hline
\end{tabular}




\begin{tabular}{|c|c|c|c|}
\hline $\begin{array}{l}\text { Los Angeles } \\
\text { Museum of } \\
\text { Holocaust }\end{array}$ & $\begin{array}{l}\text { Perspectiva temporal y simbó- } \\
\text { lico-identitaria. } \\
\text { Patrimonio Histórico y etno- } \\
\text { lógico. } \\
\text { Carácter unidisciplinar. }\end{array}$ & \begin{tabular}{|l|} 
Uso didáctico anecdótico. \\
Comunicación unidireccional. \\
Predominio de recursos pasi- \\
vos tradicionales y TIC. \\
Sin integración de contenidos \\
(solo información), salvo acti- \\
tudes en audiovisuales. \\
Sin conexiones con entorno. \\
Contextualización temporal, \\
espacial y social. \\
Finalidad academicista, pro- \\
pagandística y sociocrítica.
\end{tabular} & $\begin{array}{l}\text { Escala de identidad social, de } \\
\text { carácter histórico y etnológi- } \\
\text { co. } \\
\text { Trabaja las señas de identidad, } \\
\text { aunque a veces desde una vi- } \\
\text { sión estereotipada. } \\
\text { Tratamiento de la identidad a } \\
\text { través de vínculos afectivo- } \\
\text { emocionales. }\end{array}$ \\
\hline $\begin{array}{l}\text { Museum of } \\
\text { Tolerance }\end{array}$ & $\begin{array}{l}\text { Perspectiva fetichista y sim- } \\
\text { bólico-identitaria. } \\
\text { Patrimonio Histórico y etno- } \\
\text { lógico. } \\
\text { Carácter unidisciplinar. }\end{array}$ & $\begin{array}{l}\text { Integración didáctica plena. } \\
\text { Comunicación multidireccio- } \\
\text { nal. } \\
\text { Predominio de recursos pasi- } \\
\text { vos tradicionales y TIC, con } \\
\text { representaciones integrales. } \\
\text { Activos TIC, a través de orde- } \\
\text { nadores disponibles. } \\
\text { Integración de contenidos } \\
\text { conceptuales con actitudina- } \\
\text { les. } \\
\text { Sin conexiones con entorno. } \\
\text { Contextualización temporal, } \\
\text { espacial y social. } \\
\text { Finalidad propagandística y } \\
\text { sociocrítica. }\end{array}$ & $\begin{array}{l}\text { Escala de identidad social, de } \\
\text { carácter histórico y etnológi- } \\
\text { co. } \\
\text { Trabaja las señas de identidad, } \\
\text { aunque a veces desde una vi- } \\
\text { sión estereotipada. } \\
\text { Tratamiento de la identidad a } \\
\text { través de vínculos afectivo- } \\
\text { emocionales. }\end{array}$ \\
\hline $\begin{array}{l}\text { 9-11 Memorial } \\
\text { Museum }\end{array}$ & $\begin{array}{l}\text { Perspectiva fetichista y sim- } \\
\text { bólico-identitaria. } \\
\text { No se sigue una tipología pa- } \\
\text { trimonial tradicional, es más } \\
\text { un patrimonio social. } \\
\text { Carácter interdisciplinar. }\end{array}$ & $\begin{array}{l}\text { Uso didáctico anecdótico del } \\
\text { patrimonio. } \\
\text { Comunicación unidireccional } \\
\text { y multidireccional en charlas. } \\
\text { Predominio de recursos pasi- } \\
\text { vos tradicionales y TIC, con } \\
\text { activos tradicionales, a través } \\
\text { de charlas y debates. } \\
\text { Integración de contenidos } \\
\text { conceptuales con actitudina- } \\
\text { les. } \\
\text { Conexiones territoriales. } \\
\text { Contextualización temporal, } \\
\text { espacial y social. } \\
\text { Finalidad propagandística y } \\
\text { sociocrítica. }\end{array}$ & $\begin{array}{l}\text { Escala de identidad social. } \\
\text { Tratamiento de la identidad a } \\
\text { través de vínculos afectivo- } \\
\text { emocionales. }\end{array}$ \\
\hline $\begin{array}{l}\text { Guggenheim } \\
\text { NY }\end{array}$ & $\begin{array}{l}\text { Perspectiva estética. } \\
\text { Patrimonio Histórico-artísti- } \\
\text { co. } \\
\text { Carácter unidisciplinar. }\end{array}$ & $\begin{array}{l}\text { Sin interés socioeducativo. } \\
\text { Comunicación unidireccional. } \\
\text { Predominio de recursos pasi- } \\
\text { vos tradicionales y TIC. } \\
\text { Solo se transmite información } \\
\text { y datos. } \\
\text { Sin conexión con entorno y } \\
\text { sin contextualización. } \\
\text { Finalidad academicista y pro- } \\
\text { pagandística. }\end{array}$ & $\begin{array}{l}\text { Discurso aidentitario, desde } \\
\text { las perspectivas de las relacio- } \\
\text { nes patrimonio-identidad, tan- } \\
\text { to en lo referido a las escalas, } \\
\text { representaciones y constitu- } \\
\text { ción, aunque siempre están } \\
\text { presentes los aspectos artísti- } \\
\text { cos. }\end{array}$ \\
\hline
\end{tabular}




\begin{tabular}{|c|c|c|c|}
\hline $\begin{array}{l}\text { Metropolitan } \\
\text { Museum of } \\
\text { Art }\end{array}$ & $\begin{array}{l}\text { Perspectiva fetichista, monu- } \\
\text { mental, estética y temporal. } \\
\text { Patrimonio Histórico-artísti- } \\
\text { co. } \\
\text { Carácter unidisciplinar. }\end{array}$ & $\begin{array}{l}\text { Uso didáctico anecdótico del } \\
\text { patrimonio. } \\
\text { Comunicación unidireccio- } \\
\text { nal. } \\
\text { Predominio de recursos pasi- } \\
\text { vos tradicionales y TIC y re- } \\
\text { creaciones integrales. } \\
\text { Solo se transmite información } \\
\text { y datos. } \\
\text { Sin conexión con entorno. } \\
\text { Contextualización temporal y } \\
\text { espacial. } \\
\text { Finalidad academicista y pro- } \\
\text { pagandística. }\end{array}$ & $\begin{array}{l}\text { Discurso aidentitario, desde } \\
\text { las perspectivas de las relacio- } \\
\text { nes patrimonio-identidad, } \\
\text { tanto en lo referido a las esca- } \\
\text { las, representaciones y consti- } \\
\text { tución, aunque siempre están } \\
\text { presentes los aspectos artísti- } \\
\text { cos. }\end{array}$ \\
\hline $\begin{array}{l}\text { Museum of } \\
\text { Modern Art }\end{array}$ & $\begin{array}{l}\text { Perspectiva fetichista, monu- } \\
\text { mental y estética. } \\
\text { Patrimonio artístico. } \\
\text { Carácter unidisciplinar. }\end{array}$ & $\begin{array}{l}\text { Uso didáctico anecdótico del } \\
\text { patrimonio. } \\
\text { Comunicación unidireccional. } \\
\text { Predominio de recursos pasi- } \\
\text { vos tradicionales y TIC y acti- } \\
\text { vos tradicionales a través de } \\
\text { juegos. } \\
\text { Solo se transmite información } \\
\text { y datos. } \\
\text { Sin conexión con entorno y } \\
\text { sin contextualización de con- } \\
\text { tenidos. } \\
\text { Finalidad academicista y pro- } \\
\text { pagandística. }\end{array}$ & $\begin{array}{l}\text { Discurso aidentitario, desde } \\
\text { las perspectivas de las relacio- } \\
\text { nes patrimonio-identidad, } \\
\text { tanto en lo referido a las esca- } \\
\text { las, representaciones y consti- } \\
\text { tución, aunque siempre están } \\
\text { presentes los aspectos artísti- } \\
\text { cos. }\end{array}$ \\
\hline $\begin{array}{l}\text { Museo de El } \\
\text { Barrio }\end{array}$ & $\begin{array}{l}\text { Perspectiva estética. } \\
\text { Patrimonio artístico. } \\
\text { Carácter unidisciplinar. }\end{array}$ & $\begin{array}{l}\text { Sin interés socioeducativo. } \\
\text { Comunicación unidireccional. } \\
\text { Predominio de recursos pasi- } \\
\text { vos tradicionales y TIC. } \\
\text { Solo se transmite información } \\
\text { y datos. } \\
\text { Sin conexión con entorno y } \\
\text { sin contextualización de con- } \\
\text { tenidos. } \\
\text { Finalidad academicista y pro- } \\
\text { pagandística. }\end{array}$ & $\begin{array}{l}\text { Escala de identidad social, de } \\
\text { carácter artístico. } \\
\text { Trabaja las señas de identi- } \\
\text { dad, aunque a veces desde una } \\
\text { visión estereotipada. } \\
\text { Tratamiento de la identidad a } \\
\text { través de vínculos afectivo- } \\
\text { emocionales. }\end{array}$ \\
\hline $\begin{array}{l}\text { Museum of } \\
\text { the City of } \\
\text { New York }\end{array}$ & $\begin{array}{l}\text { Perspectiva temporal, de di- } \\
\text { versidad y simbólico-identita- } \\
\text { ria. } \\
\text { Patrimonio Histórico-artístico } \\
\text { y etnológico. Carácter inter- } \\
\text { disciplinar. }\end{array}$ & $\begin{array}{l}\text { Recurso didáctico. } \\
\text { Comunicación unidireccio- } \\
\text { nal, multidireccional en talle- } \\
\text { res. } \\
\text { Predominio de recursos pasi- } \\
\text { vos tradicionales y TIC. Acti- } \\
\text { vos tradicionales en espacios } \\
\text { en talleres. } \\
\text { Integración simple de infor- } \\
\text { mación, con procedimientos } \\
\text { en talleres. } \\
\text { Conexión territorial y contex- } \\
\text { tualización funcional, tempo- } \\
\text { ral, espacial y social. } \\
\text { Finalidad sociocrítica. }\end{array}$ & $\begin{array}{l}\text { Escala de identidad social. } \\
\text { Relación identidad con patri- } \\
\text { monio histórico, a partir de } \\
\text { una visión territorial y efecti- } \\
\text { vo-emocional. } \\
\text { Tratamiento patrimonial } \\
\text { como seña de identidad so- } \\
\text { cial. }\end{array}$ \\
\hline
\end{tabular}




\begin{tabular}{|c|c|c|c|}
\hline $\begin{array}{l}\text { National } \\
\text { Museum of } \\
\text { the American } \\
\text { Indian }\end{array}$ & $\begin{array}{l}\text { Perspectiva monumental, es- } \\
\text { tética, temporal, de diversidad } \\
\text { y simbólico-identitaria. } \\
\text { Patrimonio Histórico-artísti- } \\
\text { co, etnológico y científico- } \\
\text { tecnológico, desde visión ho- } \\
\text { lística. Carácter interdiscipli- } \\
\text { nar. }\end{array}$ & \begin{tabular}{|l|} 
Uso didáctico de carácter \\
anecdótico del patrimonio. \\
Comunicación unidireccional. \\
Predominio de recursos pasi- \\
vos tradicionales y TIC, con \\
reconstrucciones integrales. \\
Integración compleja de con- \\
tenidos. \\
Conexión interterritorial y \\
contextualización funcional, \\
temporal y espacial. \\
Finalidad academicista y so- \\
ciocrítica.
\end{tabular} & $\begin{array}{l}\text { Escala de identidad poliidenti- } \\
\text { taria, de carácter etnológico. } \\
\text { Trabaja señas de identidad. } \\
\text { Visiones afectivo-emociona- } \\
\text { les y territoriales. }\end{array}$ \\
\hline
\end{tabular}

Fuente: elaboración propia.

Los siguientes apartados presentan la discusión de los resultados, contrastando la información obtenida entre los diferentes museos seleccionados, también en función de las diferentes categorías de análisis establecidas.

\subsection{CATEGORÍA 1. CONCEPTO DE PATRIMONIO}

Al haber seleccionado una muestra de centros patrimoniales de carácter histórico, no es sorprendente que el elemento común sea el tratamiento del patrimonio histórico. Sin embargo, junto a esta tipología patrimonial, aparecen otras manifestaciones patrimoniales, fruto del tratamiento interdisciplinar que le han otorgado estas instituciones. Así, es habitual que el patrimonio etnológico aparezca representado junto al histórico en las exposiciones y materiales elaborados con una finalidad educativa en estos centros. El Musical Instrument Museum de Phoenix es un caso interesante porque aporta referentes científico-tecnológicos ligados a las manifestaciones histórico-artísticas y etnológicas, que en muchas ocasiones se perciben desde consideraciones holísticas. A pesar de todo ello, la conexión más habitual de los elementos históricos se realiza con el patrimonio artístico, que en la mayoría de las instituciones se presentan como características patrimoniales indisolubles de ámbito histórico-artístico.

La perspectiva patrimonial que encontramos es muy amplia, ya que se tienen en cuenta criterios estéticos, temporales, de diversidad cultural y simbólico-identitarios. Esto se pone en evidencia especialmente en el Heard Museum, donde se realiza un tratamiento integrado de los referentes patrimoniales ligados a diferentes culturas que han coexistido en el pasado y el presente en el mismo territorio de Arizona, analizando desde una perspectiva crítica las relaciones sociales que han tenido lugar a lo largo del tiempo. Por otro lado, en algunas ocasiones se pueden percibir algunas perspectivas ligadas a planteamientos excepcionalistas (o incluso fetichistas), como sucede en el Musical Instrument Museum, al presentar a grandes mitos de la canción. Sin embargo, en los grandes museos (Guggenheim, Metropolitan, Getty) predomina una visión monumentalista, fruto de la gran relevancia de una parte muy importante de sus colecciones.

Es necesario mencionar el diferente tratamiento de la identidad que se percibe en los diversos espacios patrimoniales analizados. Un ejemplo interesante que permite ilustrar esta situación es el caso de New York. Por un lado, podemos observar una visión de la identidad 
americana que quiere transmitirse al resto del mundo, como es el caso del 9/11 Memorial Museum, en el que se aborda la unidad de una nación con respecto a un acontecimiento traumático, presentando aparentemente una unicidad cultural y dirigido eminentemente a generar sentimientos y emociones particulares en los visitantes. Por otro lado, vemos otros casos en la misma ciudad que presentan la existencia de realidades muy distintas y diversas. Entre ellos se encuentran El Barrio Museum, centrado en la comunidad latina, el National Museum of the American Indian, que se focaliza en las comunidades indígenas americanas, o el Museum of the City, en el que se muestra la conflictividad social, económica y política de las que la ciudad de New York ha sido escenario a lo largo del tiempo, presentando una historia de la ciudad construida a través del activismo social. Una situación similar sucede en Los Ángeles si comparamos museos como el Getty o el LACMA, por un lado, con el Museum of Tolerance o el Museum of the Holocaust, por otro. Como en el caso de Nueva York, aquí podemos observar el contraste entre una visión identitaria global en la que estas ciudades se presentan como paradigma de las grandes metrópolis mundiales y una visión más particularista que enfatiza colectivos de resistencia sociocultural que ponen de manifiesto las características culturales de una comunidad específica.

En gran parte, conseguir este efecto de conexiones entre diferentes problemas y acontecimientos sociales que permiten una visión compleja de los fenómenos históricos a través del patrimonio, se consigue mediante un tratamiento interdisciplinar en el que se combinan diferentes perspectivas que actúan de manera complementaria para dar una visión lo más alejada posible de estereotipos. Esto permite centrarse en la comprensión de los procesos sociales que se han desarrollado históricamente y su sentido en el presente, aspectos básicos para desarrollar propuestas vinculadas a la educación para la ciudadanía a través de los museos. Sin embargo, existen ejemplos de un alto nivel de disciplinariedad, como sucede en el caso de Pueblo Grande Museum, donde la arqueología es el referente por excelencia de las exposiciones y materiales didácticos elaborados, sin ningún tipo de acercamiento a otras disciplinas que permitan una visión más integradora del patrimonio y su comunicación. Una situación similar puede observarse en otros museos como el Guggenheim o el MOMA, en los cuales se privilegia el arte y no se establecen relaciones con otros campos.

En resumen, observamos que en pocos museos de los que se incluyen en esta muestra el patrimonio se utiliza como un elemento social activo, y no solamente como un recurso que provee información sobre el pasado. Desde nuestra perspectiva, el patrimonio no solo sirve para entender el pasado, sino también para que el presente tenga sentido. Esta orientación coadyuva a que pueda actuarse de manera crítica en el presente a partir del pasado, con una proyección deseable en el futuro. Son estos aspectos los que permiten la constitución de una ciudadanía que valora el patrimonio como un elemento de respeto y de dinamización social.

\subsection{CATEGORÍA 2. MODELO DE COMUNICACIÓN PATRIMONIAL}

En esta categoría del estudio se abordan aquellos aspectos relacionados con los procesos educativos vinculados a la comunicación patrimonial. La mayoría de los centros que fueron seleccionados como informantes dan un sentido didáctico a sus colecciones, entendiendo la relevancia del papel educativo de estas instituciones para la formación de los ciudadanos, aunque en algunos centros esto se verifica de una manera más explícita que en otros. Por ejemplo, esto sucede de forma muy clara en el Museum of Tolerance, donde el patrimonio se articula a través de continuas propuestas educativas para transmitir 
al público las problemáticas del mundo judío y generar conciencia sobre la importancia del respeto y la tolerancia intercultural. La orientación didáctica también se puede observar en el Museum of the City of New York, donde se expone una gran cantidad de problemas sociales actuales. Por otro lado, hay casos en los que el patrimonio se percibe más desde una perspectiva anecdótica, casi como elementos que ilustran el discurso planteado en el museo. Esta visión se puede observar con cierta claridad en el Museum of Holocaust de Los Angeles, en el 9/11 Memorial Museum, en el Metropolitan Museum y en el MOMA.

En muchos centros, es habitual la existencia de salas o talleres en los que el patrimonio se aborda como un recurso para comprender ciertos contenidos que son tratados de forma temática en el discurso del museo. Así sucede en los casos del Pueblo Grande Museum y del Musical Instrument Museum; sin embargo, en diversos museos aparecen rincones o espacios dedicados específicamente a niños, en los que prima la perspectiva de proporcionar un espacio lúdico (a veces poco relacionado con la temática del museo) para que el público infantil pueda entretenerse mientras los mayores visitan de forma más tranquila la sala del museo o realizan las actividades que oferta la institución. Ejemplos de esta situación pueden encontrarse en el Getty y en el National Museum of the American Indian. Debería revisarse este tipo de propuestas, ya que tienden a aislar al público infantil, en vez de promover actividades en las que éste se integre dentro del discurso patrimonial.

Por otro lado, hay casos en los que la inclusión del patrimonio conservado en estos centros en programas educativos es poco clara. En estos casos se percibe que la intención principal de la institución museística es mostrar una colección, sin que haya un programa educativo que le proporcione un sustento didáctico. Así sucede en el Guggenheim, en el Museo de El Barrio de New York o en el Phoenix Art Museum, donde la base de la propuesta expositiva es la propia colección, sin tener en cuenta otros aspectos necesarios dentro de una programación educativa, como sería atender a las necesidades y características del público o abordar otros contenidos sociales más allá de la estricta presentación de la obra patrimonial.

En el ámbito del tipo de comunicación que se desarrolla y de los recursos empleados para ello, se comprueba cómo, en muchos casos, los museos, a pesar de presentar casi todos una tecnología avanzada (ipods, tablets, ordenadores) son escasos los que posibilitan una interacción entre el público, el museo y el patrimonio. Así se evidencian ejemplos como el del Getty Museum o el de Los Angeles Museum of the Holocaust, donde junto a las vitrinas, paneles y carteles de carácter tradicional se cuenta con dispositivos (ordenadores o ipods) con gran cantidad de información sobre las colecciones, el centro, selección de idiomas y actividades específicas. Sin embargo, se desaprovechan las posibilidades de usar estos recursos tecnológicos para una mayor dinamización, participación e interacción del público con otros visitantes, con el patrimonio y con otros centros y espacios patrimoniales más cercanos o más lejanos.

Por otro lado, siguen existiendo casos de centros donde los recursos con los que se cuentan son eminentemente pasivos y tradicionales, como Guggenheim, Metropolitan, El Barrio, MOMA, Museum of the City of NY, National Museum of American Indian, Phoenix Art Museum o Pueblo Grande Museum, lo que desemboca eminentemente en el predominio de propuestas de comunicación unidireccionales. Sin embargo, aun dentro de este grupo de museos, existen algunos ejemplos como LACMA, 9/11 Memorial Museum, Heard Museum o Musical Instrument Museum en los que, si bien predomina el uso de recursos de carácter tradicional, es común la existencia de talleres que permiten una dinamización de las actividades para trabajar con el patrimonio, pudiéndose llegar a 
desarrollar propuestas multidireccionales en este sentido. Sin embargo, el mejor ejemplo de propuestas multidireccionales lo hemos encontrado en el Museum of Tolerance, en donde se desarrolla una variedad de actividades educativas a través de diversos dispositivos, incluyendo juegos de rol y simulación.

Con respecto a los contenidos que se presentan en estas instituciones patrimoniales, predomina la transmisión de información ligada a las características de las colecciones que conserva cada uno de los museos. Son escasos los centros que trabajan con el público, procedimientos o técnicas patrimoniales, básicamente aquellos que desarrollan talleres vinculados a sus colecciones (Heard Museum o Pueblo Grande Museum) o aquellos museos donde los valores tienen un componente fundamental por las temáticas tratadas (Museum of Tolerance, Los Angeles Museum of the Holocaust, 9/11 Memorial Museum, National Museum of the American Indian). Lo más común es que estos contenidos se contextualicen a nivel espacial y temporal, siendo mucho menos frecuente una contextualización social. En este sentido se pueden destacar el Getty, el Heard Museum, el Musical Instrument Museum o el Museum of the City of New York, al abordar el sentido social de determinadas obras a través de cartelas y del uso de ipods.

El predominio de la transmisión, casi en exclusiva en muchos casos, de datos, conceptos e informaciones sobre los temas fundamentales de cada colección provoca que la comunicación tenga un carácter demasiado academicista. Sin embargo, destaca en algunos de ellos una finalidad propagandística en la que subyace la intencionalidad de mostrar la importancia del museo a través de la relevancia histórico-artística de las piezas que conserva y, al mismo tiempo, la relevancia de los Estados Unidos como sociedad que ha sido capaz de acumular el patrimonio de otros muchos lugares del mundo (al igual que hicieron los grandes museos nacionales europeos en los siglos XVIII y XIX). Se percibe esta visión propagandística en los grandes museos como el Getty, LACMA, Guggenheim, Metropolitan o MOMA, lo cual los diferencia de museos de carácter más sociocrítico como el Museum of Tolerance, Los Angeles Museum of Holocaust, Museum of the City of New York, National Museum of the American Indian o Heard Museum.

Debido a las características de las colecciones de algunos de estos museos, compuestas por elementos patrimoniales de gran diversidad de culturas y países, se percibe una visión interterritorial en la que se pretende analizar y explicar las características socioculturales de esas diferentes civilizaciones tratadas. Esta tendencia se puede observar en el Heard Museum, así como en el Getty, el LACMA (con múltiples ejemplos de referentes patrimoniales de origen europeo), el National Museum of the American Indian (en el caso de las diferentes culturas indígenas existentes en el continente americano) o en el Musical Instrument Museum (estructurándose las salas en función de los continentes y de las áreas de influencia musicales de cada ámbito territorial).

\subsection{CATEGORÍA 3. PATRIMONIO E IDENTIDAD}

Esta tercera categoría analiza las relaciones existentes entre los diferentes elementos patrimoniales existentes en los museos estudiados y los componentes identitarios que pueden vincularse a las diferentes sociedades y culturas que se encuentran presentes en las propuestas educativas desarrolladas en dichos centros.

En diversos museos analizados se comprueba que la identidad no es un aspecto directamente tratado en sus propuestas educativas y expositivas. De hecho, se podría decir 
que el tratamiento de la dimensión identitaria que recibe el patrimonio conservado en estas instituciones está ausente. Es decir, el patrimonio se presenta aparentemente de forma aséptica, sin pretender otorgarle un valor simbólico como elemento característico de una determinada cultura. Los ejemplos más claros de estas situaciones se pueden observar en el Getty Museum, el LACMA, el Guggenheim, el Metropolitan, el MOMA y el Phoenix Art Museum.

En otros casos, se percibe la conexión del patrimonio con las identidades a través de unos lazos afectivo-emocionales, fruto de su concepción como símbolo que representa su relación con unos acontecimientos o referentes de carácter histórico. Estas situaciones se pudieron observar en el Museum of Tolerance, Los Angeles Museum of Holocaust, el 9/11 Memorial Museum y Pueblo Grande Museum, así como en centros vinculados a tradiciones populares como el Museo del Barrio de New York y el Musical Instrument Museum de Phoenix.

Por otro lado, existen algunos ejemplos en los que las conexiones con la identidad se realizan a través de informaciones y artefactos de carácter histórico y/o territorial que caracterizan a determinadas comunidades, por lo que el sentido de identidad se promueve a través de vínculos sociales. A veces, estos vínculos se refieren a identidades locales, como por ejemplo en el Museo de El Barrio de New York y el Museum of the City of New York. En otras ocasiones, los vínculos pueden llegar a percibirse desde perspectivas multi-identitarias, como podría entenderse en el caso de National Museum of the American Indian, a través de las conexiones entre las diferentes civilizaciones indígenas del continente americano, o en el Heard Museum, al interconectar diferentes identidades de diversas sociedades dentro de un mismo territorio.

\section{CONCLUSIONES}

En este trabajo hemos examinado las características y tipologías patrimoniales que se abordan en los procesos educativos desarrollados en quince museos estadounidenses (cuatro de Arizona, cuatro de California, y siete de Nueva York), poniendo especial atención en las relaciones entre patrimonio e identidad, desde la perspectiva de la educación para la ciudadanía. Los datos fueron recabados a través de observaciones directas en cada museo. Para el análisis de los datos hemos utilizado tres categorías: a) el concepto de patrimonio, b) el modelo de comunicación patrimonial, y c) la relación entre patrimonio e identidad. Nos interesaba particularmente explorar la manera en que algunas cuestiones que entendemos básicas en la formación para la ciudadanía (identidad, problemas sociales relevantes, conexión con el entorno) son atendidas en ellos a través de las propuestas de educación patrimonial.

Como se ha detallado en las secciones anteriores, en nuestra muestra hemos detectado un amplio espectro de situaciones. En un extremo, hemos encontrado instituciones patrimoniales que tienen una visión bastante tradicional y academicista, centrándose en presentar una perspectiva aparentemente aséptica del patrimonio, como si este fuera un elemento externo a las relaciones socioculturales del pasado y de la actualidad. En esta situación encontramos casos como Phoenix Art Museum, Getty, LACMA, Guggenheim, MOMA o Metropolitan (grandes museos que responden fundamentalmente a una tipología patrimonial artística). En el otro extremo hemos identificado museos cuyas propuestas de 
Estudios Pedagógicos XLIII, N ${ }^{\circ}$ : 29-48, 2017

EDUCACIÓN PARA LA CIUDADANÍA E IDENTIDAD EN LOS MUSEOS DE ESTADOS UNIDOS. ANÁLISIS DESDE LA PERSPECTIVA DE LA EDUCACIÓN PATRIMONIAL

trabajo centran su atención en los problemas de la sociedad en la que se inserta la institución, incluyendo su contextualización en el territorio y los referentes multiidentitarios, como Museum of Tolerance, Los Angeles Museum of Holocaust, Museum of the City of New York o Heard Museum.

Esta diferencia puede explicarse fundamentalmente por la concepción del patrimonio y de la educación patrimonial de los gestores culturales responsables de la comunicación en estas instituciones, tal como ya se ha puesto de manifiesto en otros estudios (MartínCáceres \& Cuenca, 2011, 2016). Una concepción próxima a perspectivas patrimoniales y educativas de carácter sociocrítico promueve el desarrollo de propuestas abiertas, participativas, dinámicas e interactivas, independientemente, en muchos casos, de los medios de que se dispongan para ello.

En esta misma línea, se puede destacar que una gran parte de los grandes museos que se han analizado (Getty, LACMA, Guggenheim, MOMA, Metropolitan), si bien cuentan con unas colecciones excepcionales, no se han caracterizado por propuestas didácticas cercanas al tratamiento de la ciudadanía o que atiendan al análisis de problemas sociales del pasado o del presente. Por otro lado, los museos de sitio, de localidad o comunitarios, a pesar de tener menos recursos, son los que conectan más el patrimonio con los aspectos más cercanos a la ciudadanía. Podríamos decir que la interdisciplinariedad en el tratamiento de los contenidos de los museos es un elemento clave para desarrollar propuestas que trabajen la ciudadanía, la identidad y los problemas sociales (Heard Museum, 9/11 Memorial Museum, Museum of the City of New York y National Museum of American Indian).

No se constataron diferencias en el tratamiento del patrimonio en relación con la educación en estos centros analizados en función del estado o localidad en el que se sitúan. Como hemos dicho, estas diferencias se relacionan con las características del museo y de la tipología patrimonial que se aborda en cada institución. Los museos que tienen una temática unidisciplinar (históricos o artísticos) tienden a tener una visión más académica, mientras que en los que abordan temáticas más amplias y reflejan problemas sociales pasados o presentes (como son los casos del Museum of Tolerance y de Los Angeles Museum of Holocaust) el tratamiento de la ciudadanía vinculada a estos problemas y a las identidades se hace más evidente.

Finalmente, en este estudio también observamos que el uso de tecnologías modernas en la educación patrimonial no es una condición necesaria para el tratamiento de la ciudadanía y las propuestas didácticas sobre sus problemas asociados. La tecnología es un instrumento que puede facilitar la conexión y la dinamicidad, pero depende de la perspectiva desde la que se emplee. Por ejemplo, algunos museos como Guggenheim, Getty o LACMA utilizan tecnologías muy actuales (ipods o aplicaciones disponibles por WIFI, por ejemplo), pero sus propuestas didácticas -tanto en lo que respecta a estrategias y a contenidos- son bastantes tradicionales, quizás sin sacar provecho de toda la potencialidad que estos dispositivos podrían ofrecer. Regresando a Duncan (1994), cuestionarse lo que vemos y lo que no vemos en un museo -incluyendo la pregunta de qué autoridad decide lo que se presenta y cómo se presenta- nos remite a interrogarnos quién constituye la comunidad, y quiénes deberían ejercer el poder para definir su identidad. Como bien observa Aronsson (2014), los museos son espacios en los cuales la definición de identidad está siendo negociada permanentemente. Nuestro trabajo refleja una fotografía sobre lo que hacen quince museos de diferente tamaño y orientación en torno al patrimonio y la identidad en un momento histórico determinado. Para entender mejor la película y capturar las dinámicas 
de continuidad y cambio en función de factores internos y externos, sería pertinente repetir este estudio con los mismos museos dentro de una década.

Por otro lado, las líneas de estudio que se desarrollan en un futuro próximo, dentro del proyecto de investigación en el que estamos inmersos, abordan el análisis de las conexiones entre los tópicos abordados en este trabajo (patrimonio, identidad, ciudadanía) y el papel que las inteligencias emocionales (Extremera \& Fernández-Berrocal, 2003) y territorial (Girardot \& Miedes, 2008) juegan dentro del campo educativo y fundamentalmente en el caso de la educación reglada.

\section{REFERENCIAS BIBLIOGRÁFICAS}

Álvarez-Gayou, J. L. (2003). Cómo hacer investigación cualitativa. Fundamentos y metodología. México D.F.: Paidós Educador.

Aronsson, P. (2014). Explaining national museums. In S. Knell, P. Aronsson \& A. Amundsen (Eds.), National museums: New studies from around the world (pp. 29-54). New York: Routledge.

Brusa, A. (2011). Paisaje y patrimonio, entre búsqueda, formación y ciudadanía. Her\&mus: Heritage and Museography, 7, 80-84.

Calaf, R. (2010). Un modelo de investigación en didáctica del patrimonio. Enseñanza de las Ciencias Sociales, 9, 17-27.

Calaf, R., \& Suárez, M. A. (2015). Acción educativa en museos. Su calidad desde la evaluación cualitativa. Gijón: Trea.

Copeland, T. (2009). Archaeological heritage education: citizenship from the ground up. Treballs d'Arqueologia, 15, 9-20.

Cuenca, J. M. (2002). El patrimonio en la didáctica de las ciencias sociales. Análisis de concepciones, dificultades y obstáculos para su integración en la enseñanza obligatoria. Huelva: Universidad de Huelva. Recuperado de http://rabida.uhu.es/dspace/handle/10272/2648

Cuenca, J. M., \& Estepa, J. (2013). La educación patrimonial: líneas de investigación actual y nuevas perspectivas. En J. Estepa (Ed.), La educación patrimonial en la escuela y el museo: investigación y experiencias (pp. 343-355). Huelva: Universidad de Huelva.

Cuenca, J. M., \& Martín-Cáceres, M. J. (2014). Manual para el desarrollo de proyectos educativos de museos. Gijón: Trea.

Cuenca, J. M., Martín-Cáceres, M. J., Ibáñez, A., \& Fontal, O. (2014). La educación patrimonial en las instituciones patrimoniales españolas. Situación actual y perspectivas de futuro. Clío. History and History Teaching, 40. Recuperado de http://clio.rediris.es/n40/articulos/mono/ MonCuencaetal2014.pdf

Davis, P. (2007). Place exploration: museums, identity, community. In S. Watson (Ed.), Museums and their communities (pp. 53-75). Abingdon \& New York: Routledge.

Duncan, C. (1994). Art museums and the ritual of citizenship. In S. M. Pearce (Ed.), Interpreting Objects and Collections (pp. 279-286). London-New York: Routledge.

Estepa, J. (2013). La educación patrimonial en la escuela y el museo: investigación y experiencias. Huelva: Universidad de Huelva.

Extremera, N., \& Fernández-Berrocal, P. (2003). La inteligencia emocional en el contexto educativo: hallazgos científicos de sus hallazgos en el aula. Revista de educación, 332, 97-116. doi:10.4438/1988-592X-0034-8082-RE

Falk, J. H. (2016). Identity and the museum visitor experience. London: Routledge.

Falk, J. H., Dierking, L. D., \& Adams, M. (2011). Living in a learning society: museums and free-choice learning. In S. Macdonald (Ed.), A companion to museum studies (pp. 323-339). Chichester: Wiley-Blackwell. 
Estudios Pedagógicos XLIII, N 4: 29-48, 2017

EDUCACIÓN PARA LA CIUDADANÍA E IDENTIDAD EN LOS MUSEOS DE ESTADOS UNIDOS. ANÁLISIS DESDE LA PERSPECTIVA DE LA EDUCACIÓN PATRIMONIAL

Flick, U. (2004). Introducción a la investigación cualitativa. Madrid: Morata.

Fontal, O. (2013). La educación patrimonial: del patrimonio a las personas. Gijón: Trea.

Fontal, O., \& Gómez-Redondo, C. (2016). Heritage Education and Heritagization Processes: SHEO Methodology for Educational Programas Evaluation. Interchange, 47(1), 65-90. doi:10.1007/ s10780-015-9269-z

Fontal, O., \& Ibáñez, A. (2015). Estrategias e instrumentos para la educación patrimonial en España. Educatio Siglo XXI, 33(1), 15-32. doi:10.6018/j/222481

Fontal, O., \& Ibáñez, A. (2017). La investigación en educación patrimonial. Evolución y estado actual a través del análisis de indicadores de alto impacto. Revista de Educación, 375, 184-214. doi:10.4438/1988-592X-RE-2016-375-340

Gibbs, G. R. (2012). Análisis de datos cualitativos. Madrid: Morata.

Girardot, J., \& Miedes, B. (2008). International Conference of Territorial Intelligence. Huelva: CAENTI.

Ibáñez, A., Asensio, M., Vicent, N., \& Cuenca, J. M. (2012). Mobile devices: a tool for tourism and learning at archaeological sites. International Journal of Web Based Communities, 8(1), 57-72. doi:10.1504/IJWBC.2012.044682

Jiménez, R., Cuenca, J.M., \& Ferreras, M. (2010). Heritage education: Exploring the conceptions of teachers and administrators from the perspective of experimental and social science teaching. Teaching and Teacher Education, 26(6), 1319-1331. doi:10.1016/j.tate.2010.01.005

León, O. G., \& Montero, I. (2002). Métodos de investigación en Psicología y Educación. Madrid: Mc Graw Hill.

Macdonald, S. (2011). A companion to museum studies. Chichester, UK: Wiley-Blackwell.

Martín-Cáceres, M. J. (2012). La educación y la comunicación patrimonial. Una mirada desde el Museo de Huelva. Huelva: Universidad de Huelva. Recuperado de http://rabida.uhu.es/dspace/ handle/10272/6048

Martín-Cáceres, M. J., \& Cuenca, J. M. (2011). La enseñanza y el aprendizaje del patrimonio en los museos: la perspectiva de los gestores. Revista de Psicodidáctica, 16(1), 99-122.

Martín-Cáceres, M. J., \& Cuenca, J. M. (2015). Educomunicación del patrimonio. Educatio Siglo XXI, 33(1), 33-54. doi:10.6018/j/222491

Martín-Cáceres, M. J., \& Cuenca, J. M. (2016). Communicating heritage in museums: outlook, strategies and challenges through a SWOT. Museum Management and Curatorship, 31(3), 299 316. doi:10.1080/09647775.2016.1173576

Molina, S., Llonch, N., \& Martínez, T. (2016). Identidad, ciudadanía y patrimonio. Educación histórica para el siglo XXI. Gijón: Trea.

Pinto H. (2016). Educação Histórica e Patrimonial: conceções de alunos e professores sobre o passado em espaços do presente. Universidade do Minho. Porto: CITCEM.

San Fabián, J. L. (2014). Evaluar programas socioeducativos en tiempos de crisis: un enfoque organizacional. Oviedo: Trabe.

Schugurensky, D. (2016). Educación ciudadana e identidad: La disputa por la comunidad imaginada. En R. López Facal (Ed.), Ciencias sociales, educación y futuro. Investigaciones en didáctica de las ciencias sociales (pp. 48-61). Santiago de Compostela: Universidad de Santiago de Compostela.

Semedo, A. (2007). Museus, educação e cidadania. Actas Conferência Museus e Sociedade (pp. 2535). Caminha: Museu Municipal de Caminha-Câmara Municipal de Caminha.

Semedo, A. (2015). Representações e identidade em exposições de museus. Clío. History and History Teaching, 41. Recuperado de http://clio.rediris.es/n41/articulos/mono/MonAsemedo2015.pdf

Vicent, N., Ibáñez, A., \& Asensio, M. (2015). Evaluación de programas de educación patrimonial de base tecnológica. Virtual Archaeology Review, 6(13), 18-25. doi:10.4995/var.2015.4367

Wiersma, W. (2000). Research Methods in Education. An Introduction. Boston: Allyn \& Bacon. 